\title{
A Study of General AC Machine Modeling with Matrix Vector Using DQ Transformation
}

\author{
Sun-Ki Hong*
}

\begin{abstract}
$\mathrm{AC}$ machines are in wide use in industry and $d-q$ transformation from 3 phase of a, b, c is commonly used to analyze these kinds of machines. The equivalent circuits of $\mathrm{d}$ and $\mathrm{q}$ axis are, however, generally cross coupled and difficult to analyze. In this study, a modeling technique of AC machine including induction and PM synchronous motors using matrix vector is proposed. With that model, it can not only explain the AC machines physically but also make it simple to analyze them. The separating process of $\mathrm{d}$ and $\mathrm{q}$ components is not needed in this model and this model can be applied to analyze asymmetric motors like IPMSM machine. With this technique, the model becomes simple, easy to understand physically, and yields results that are the same as those from other models. These simulation results of the proposed model for induction motor are compared with those of other models to verify the method proposed.
\end{abstract}

Key Words : AC Machine, $d-q$ Transformation, Complex Vector, Matrix Vector, Asymmetric Machine

\section{Introduction}

AC machines including induction machines and synchronous machines are in wide used in industrial fields. The $\mathrm{d}^{-}$qtransformation is commonly used to easily analyze machine features [1-5]. However, the equivalent circuits of the $d$ and $q$ axis are generally cross coupled, making it difficult to calculate the features. A model using complex vector [5] does not

\footnotetext{
* Main author : Department of Digital Control Eng., Hoseo University, Korea

Tel : +82-41-540-5674, Fax : +82-41-540-5587

E-mail : skhong@hoseo.edu

Date of submit : 2013. 6.13.

First assessment : 2013. 6. 6 .

Completion of assessment : 2013. 7.24.
}

have a cross coupled form and looks simple for understanding the flow, but it is difficult to apply this model to asymmetric machines like IPMSM. In this study, a modeling technique of AC machine including induction and PMSM using matrix vector is proposed and can explain the $\mathrm{AC}$ machine physically. This model does not need a separating process for $\mathrm{d}$ and q components. With this method, the model becomes simple and easy to understand physically, and can yield results the same as those from the other models. These simulation results of the proposed model are compared with those of other models to verify the proposed method. 


\section{Conventional Induction Motor (IM) Model}

\subsection{IM model using $\psi=\omega_{b} \lambda$ at arbitrary reference frame}

The $\mathrm{d}^{-\mathrm{q}}$ voltage equations of stator and rotor from 3 phases become (1), (2) [4]. (3), (4) shows the magnetic flux linkage and torque equations.

$$
\begin{aligned}
& v_{q s}=P \lambda_{q s}+\omega \lambda_{d s}+r_{s} i_{q s} \\
& v_{d s}=p \lambda_{d s}+\frac{\omega}{\omega_{b}} \lambda_{q s}+r_{s} i_{d s} \\
& v_{q r}^{\prime}=p \lambda_{q r}^{\prime}+\left(\omega-\omega_{r}\right) \lambda_{d s}^{\prime}+r_{r}^{\prime} i_{q r}^{\prime} \\
& v_{d r}^{\prime}=p \lambda_{d r}^{\prime}+\left(\omega-\omega_{r}\right) \lambda_{q s}^{\prime}+r_{r}^{\prime} i_{d r}^{\prime}
\end{aligned}
$$

$$
\left[\begin{array}{c}
\lambda_{q s} \\
\lambda_{d s} \\
\lambda_{q r}^{\prime} \\
\lambda_{d r}^{\prime}
\end{array}\right]=\left[\begin{array}{ccccc}
L_{b}+L_{m} & 0 & L_{m} & 0 \\
0 & L_{b}+L_{m} & 0 & L_{m} \\
L_{m} & 0 & L_{k}+L_{m} & 0 \\
0 & L_{m} & 0 & L_{k}+L_{m}
\end{array}\right]\left[\begin{array}{c}
i_{q s} \\
i_{d s} \\
i_{q r}^{\prime} \\
i_{d r}^{\prime}
\end{array}\right]
$$

$$
\begin{aligned}
T_{e m} & =\frac{3}{2} \frac{P}{2}\left(\lambda_{d s} i_{d s}-\lambda_{q s} i_{d s}\right) \\
& =\frac{3}{2} \frac{P}{2} L_{m}\left(i_{d r}^{\prime} i_{q s}-i_{q r}^{\prime} i_{d s}\right)
\end{aligned}
$$

Where, $\mathrm{p}$ is the differential operator, $\mathrm{P}$ is number of poles, $\omega$ is the speed of frame and $\omega_{r}$ is the speed of rotor, $\lambda$ is flux linkage, $L_{l s}, L_{l r}$ are leakage inductance, $T_{\text {emis }}$ developed motor torque and means the transferred value to stator side. From these equations, Ong [4] adopted the flux linkage per sec $\psi=\omega_{b} \lambda$, in which dimension is voltage for convenience. From this idea, (1) (4) transformed to (5) (8) [4] and the flux linkage equation (3) is changed to flux linkage per second equation (7).

$$
\begin{aligned}
& v_{q s}=\frac{p}{\omega_{b}} \psi_{q s}+\frac{\omega}{\omega_{b}} \psi_{d s}+r_{s} i_{q s} \\
& v_{d s}=\frac{p}{\omega_{b}} \psi_{d s}+\frac{\omega}{\omega_{b}} \psi_{q s}+r_{s} i_{d s} \\
& v_{q r}^{\prime}=\frac{p}{\omega_{b}} \psi_{q r}^{\prime}+\left(\frac{\omega-\omega_{r}}{\omega_{b}}\right) \psi_{d s}^{\prime}+r_{r}^{\prime} i_{q r}^{\prime} \\
& v_{d r}^{\prime}=\frac{p}{\omega_{b}} \psi_{d r}^{\prime}+\left(\frac{\omega-\omega_{r}}{\omega_{b}}\right) \psi_{q s}^{\prime}+r_{r}^{\prime} i_{d r}^{\prime}
\end{aligned}
$$

$\left[\begin{array}{l}\psi_{q s} \\ \psi_{d s} \\ \psi_{q r}^{\prime} \\ \psi_{d r}^{\prime}\end{array}\right]=\left[\begin{array}{cccc}x_{b}+x_{m} & 0 & x_{m} & 0 \\ 0 & x_{b}+x_{m} & 0 & x_{m} \\ x_{m} & 0 & x_{b}+x_{m} & 0 \\ 0 & x_{m} & 0 & x_{k}+x_{m}\end{array}\right]\left[\begin{array}{l}i_{q s} \\ i_{d s} \\ i_{q r}^{\prime} \\ i_{d r}^{\prime}\end{array}\right]$

$T_{e m}=\frac{3}{2} \frac{p}{2 \omega_{b}}\left(\psi_{d s} i_{q s}-\psi_{q s} i_{d s}\right)$

Fig. 1 shows the block diagram of IM with Matlab Simulink. The $\mathrm{d}^{-\mathrm{q}}$ voltage equations are transformed from 3 phase voltage equations and the differential terms for $p \psi$ in (5), (6) are used to make IM block. Fig. 2 shows the q-axis block in Fig. 1 [1]. Fig. 1 appears simple however, $\psi$ is used to erase the variable $i_{q d}$ and the block requires the complex equation block of Fig. 2. In addition, $\psi$ does not appear to have clear physical meaning.

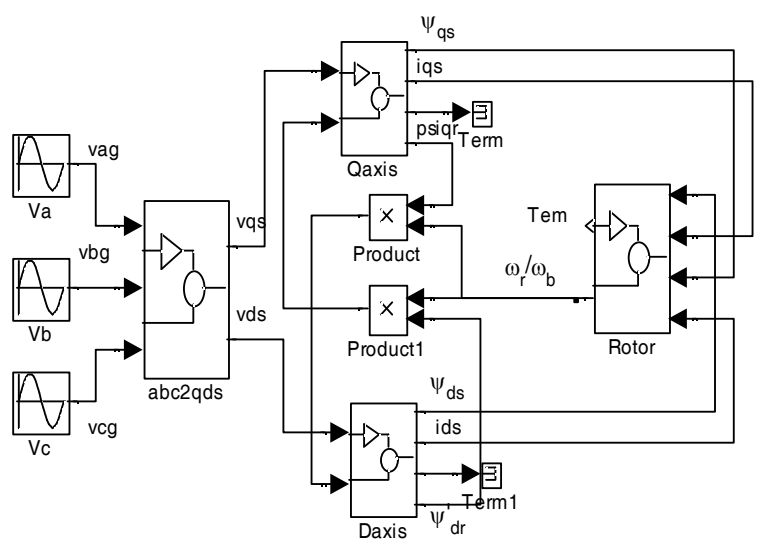

Fig. 1. IM model using $\psi=\omega_{b} \lambda$ for arbitrary reference frame 


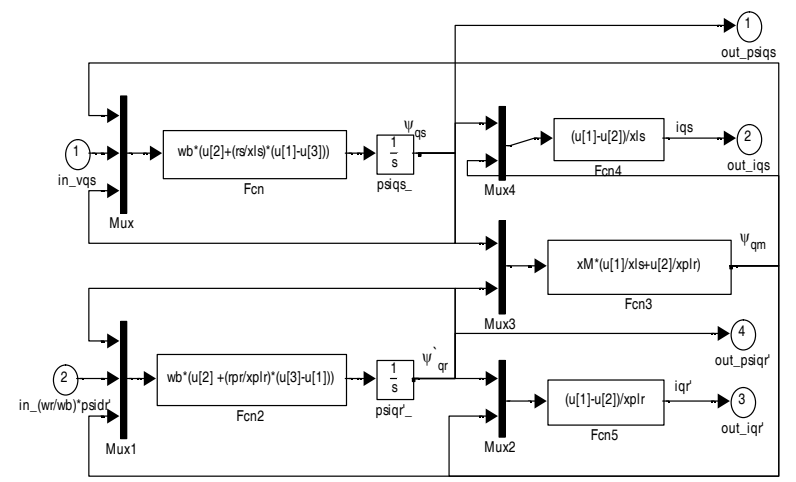

Fig. 2. Q-axis calculation block in Fig. 1

In the figure, $1 / \mathrm{s}$ means integral symbol, $\mathrm{u}[\mathrm{i}]$ is i-th input.

\subsection{Induction motor model using flux linkage $\lambda$}

As mentioned in $2.1, \psi$ model needs somewhat complex mathematical equations and has physical ambiguities in the model block. In this section, the flux linkage $\lambda$ itself is not modified but used directly to derive the integration form equations as (9) - (12) for physical meaning.

$i_{q s}=\frac{1}{L_{l s}+L_{m}} \int\left(v_{q s}-r_{s} i_{q s}-\omega \lambda_{d s}-L_{m} \frac{d}{d t} i_{q r}^{\prime}\right) d t$

$i_{d s}=\frac{1}{L_{L s}+L_{m}} \int\left(v_{d s}-r_{s} i_{d s}-\omega \lambda_{q s}-L_{m} \frac{d}{d t} i_{d r}^{\prime}\right) d t$

$i_{q r}^{s}=\frac{1}{L_{l r}^{\prime}+L_{m}} \int\left(-r_{r}^{\prime} i_{q r}^{\prime}-\left(\omega-\omega_{r}\right) \lambda_{d r}^{\prime}-L_{m} \frac{d}{d t} i_{q s}\right) d t$

$i_{d r}^{\prime}=\frac{1}{L_{l r}^{\prime}+L_{m}} \int\left(-r_{r}^{\prime} i_{d r}^{\prime}-\left(\omega-\omega_{r}\right) \lambda_{q r}^{\prime}-L_{m} \frac{d}{d t} i_{d s}\right) d t$

$\left[\begin{array}{l}\lambda_{q s} \\ \lambda_{d s} \\ \lambda_{q r}^{\prime} \\ \lambda_{d r}^{\prime}\end{array}\right]=\left[\begin{array}{ccccc}L_{k}+L_{m} & 0 & L_{m} & 0 \\ 0 & L_{k}+L_{m} & 0 & L_{m} \\ L_{m} & 0 & L_{k}+L_{m} & 0 \\ 0 & L_{m} & 0 & L_{k}+L_{m}\end{array}\right]\left[\begin{array}{c}i_{q s} \\ i_{d s} \\ i_{q r}^{\prime} \\ i_{d r}^{\prime}\end{array}\right]$

$$
T_{\varepsilon m}=\frac{3}{2} \frac{p}{2} L_{m}\left(i_{q s}^{s} i_{d r}^{s}-i_{d s}^{s} i_{q r}^{s}\right)
$$

Fig. 3 shows the block diagram made with (9) -(12) using Simulink, where $L_{s}=L_{l s}+L_{m}$, $L_{r}=L_{l r}^{\prime}+L_{m}$. This model is derived using $\lambda$, which, despite seeming slightly more complex, has physical meaning. In fact, it does not have sub-blocks, making it simpler than the previous model of using $\psi=\omega_{b} \lambda$

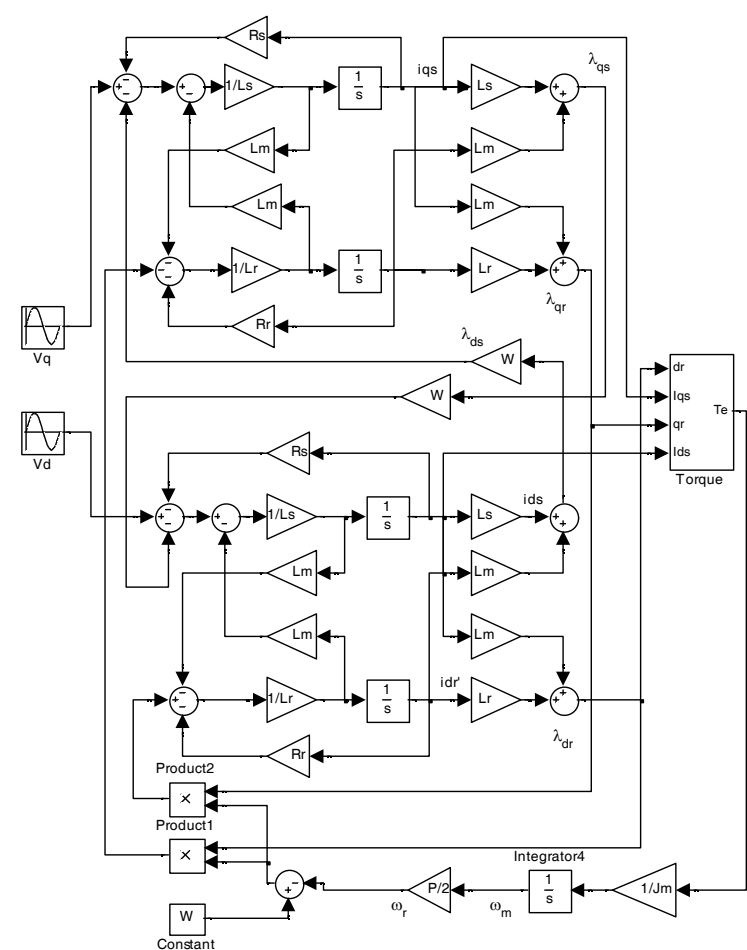

Fig. 3. IM model using flux linkage $\lambda$ for arbitrary ref. frame

\subsection{Induction motor model using complex vector}

Complex vector has areal and imaginary axis. In IM or AC machine modeling, $\mathrm{q}^{-}$axis is the real and $\mathrm{d}$-axis is the imaginary axis. This kind of modeling is simpler than the common $\mathrm{d}^{-} \mathrm{q}$ transformation 
method because it does not need to separate the $d$ and $\mathrm{q}$ components and there are no couplings between the $\mathrm{d}$ and q equivalent circuit or differential equations. With the Simulink tool, it is easy to realize to a complex vector model and the complex calculation is possible in the model block. For example, the voltage of $\mathrm{d}^{-} \mathrm{q}$ can be expressed as $v_{q d}=v_{q}+j v_{d}$, where the character in bold means complex vector. The following equations (13) and (14) show the voltage and flux linkage equations of the complex vector form in stator and rotor [5].

$v_{q d}=r_{s} i_{q d}+\frac{d}{d t} \lambda_{q d}+j \omega \lambda_{q d}$

$v_{q d r}=r_{s} i_{d s}+\frac{d}{d t} \lambda_{q d}+j \omega \lambda_{q d}$

$\lambda_{q d}=L_{s} i_{q d}+L_{m} i_{q d r}$

$\lambda_{q d r}=L_{r} i_{q d r}+L_{m} i_{q d}$

Through this approach, the current complex vector ${ }^{i}$ qd and magnetic flux linkage complex vector $\lambda_{q d r}$ become the variables to solve therefore ${ }^{i}{ }_{q d}$ and $\lambda_{q d r}$ need to be erased. With (14), the following equation of (15) is derived.

$\lambda_{q d}=\sigma L_{s} i_{q d}+\frac{l_{m}}{L_{r}} \lambda_{q d r}$

$\sigma=1-\frac{L_{m}^{2}}{L_{s} l_{r}}$

Where $\sigma$ is defined as (15) for simplicity. (15) is substituted for (13) and the following differential equations are derived.

$$
\begin{aligned}
& \sigma \frac{d}{d t} i_{q d}=v_{q d}-\left(r_{s}+j \omega \sigma L_{s}\right) i_{q d} \\
& -\frac{L_{m}}{L_{r}}\left(\frac{d}{d t} \lambda_{q d r}+j \omega \lambda_{q d r}\right)
\end{aligned}
$$

$$
\frac{d}{d t} \lambda_{q d r}=-\frac{r_{r}}{L_{r}}-j\left(\omega-\omega_{r}\right) \lambda_{q d r}+r_{r} \frac{l_{m}}{L_{r}} i_{q d}
$$

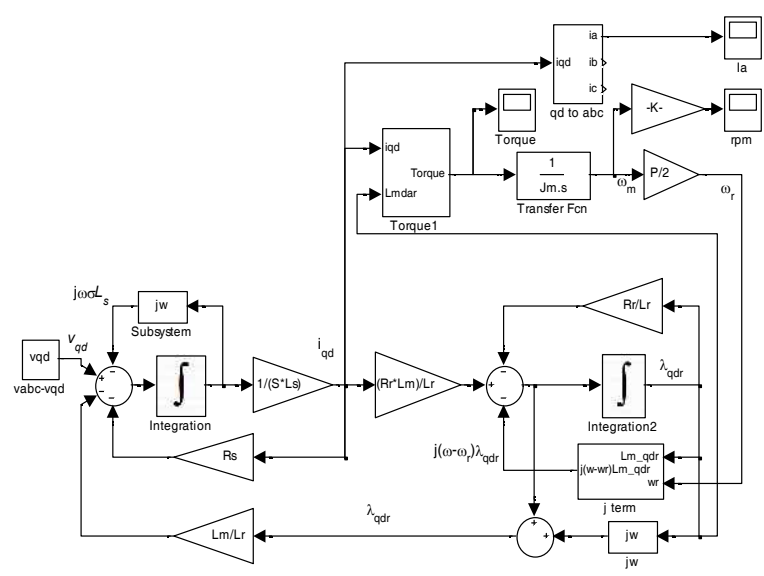

Fig. 4. IM model using complex vector for arbitrary ref. frame

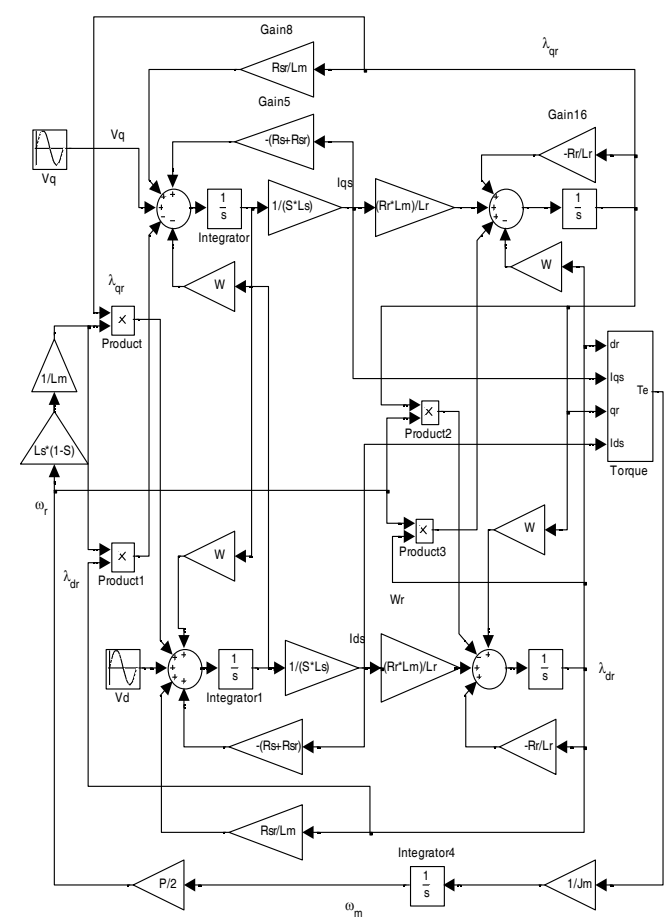

Fig. 5. IM model using scalar for arbitrary ref. frame

Fig. 4 shows the IM model derived from (16) and (17). As canbe seen in Fig. 4, this model does not need extra parameters as in $\psi$ and does not appear 
as complex as the flux linkage model in Fig. 3. Each icon has a physical meaning and is easily realized as the model in Fig. 4. In the figure, the integral symbol is a sub block that has two integral processes of each $\mathrm{q}$ and $\mathrm{d}$ axis equation. After the integrations, they are combined again to make complex vector.

Fig. 5 shows the IM model with separated differential equations from (16) and (17) [5]. The separated equations would be similar with (9) (11) and the $\mathrm{d}$ and $\mathrm{q}$ components are cross ${ }^{-}$coupled, as can be seen in Fig. 5, which makes it difficult to understand the flow, debug problems if any or analyze operations.

With these results, the IM modeling, with complex vector and $\mathrm{d}^{-\mathrm{q}}$ transformation, has a simple structure, is easy to debug and facilitates analysis of IM characteristics.

\section{Proposed Matrix Vector Model for AC Machine}

The previous complex vector model of IM is very useful to analyze induction machine. Similarly, $d^{-}-\mathrm{q}$ vector components can be expressed in the matrix form of (18).

$f_{q d}=\left[\begin{array}{l}f_{q} \\ f_{d}\end{array}\right]=f_{q}-j f_{d}$

The complex number operator is replaced with bold $\mathrm{j}$ where $\mathrm{j}$ is not just an imaginary symbol but has the matrix vector form of (19). (19) represents a $90^{\circ}$ rotation matrix whose function is the same as the complex number $j$ in the complex vector model.

$$
\boldsymbol{j}=\left[\begin{array}{cc}
0 & 1 \\
-1 & 0
\end{array}\right]
$$

\subsection{Induction motor model of matrix vector}

The basic voltage equations are almost the same with (13) and (14) except $\mathrm{j}$ is replaced with $\mathrm{j}$. In addition, every component must be expressed in matrix form. For example, the self inductance and is expressed as (20) and (21).

$\boldsymbol{L}_{s}=\left[\begin{array}{cc}L_{q} & 0 \\ 0 & L_{d}\end{array}\right]$

$\boldsymbol{\sigma}=I-L_{s}^{-1} L_{m} L_{r}^{-1} L_{m}$

The result form of the induction motor with matrix vector does not differ greatly from Fig. 4.

\subsection{Asymmetric PMSM model of matrix vector}

Permanent magnets and magnetic asymmetry are the differences between IM and asymmetric PMSM. The asymmetric component in asymmetric PMSM cannot be expressed with complex vector model. However, the asymmetric component can be expressed via a matrix model as in (22).

$\boldsymbol{L}_{s}=\left[\begin{array}{cc}L_{q} & L_{q d} \\ L_{d q} & L_{d}\end{array}\right]$

The asymmetric machine, like the interior type permanent magnet synchronous machine, can be analyzed with complex vector form.

Eq. (23) (26) shows the AC machines' stator, rotor, and field equations where bold characters refer not to a complex but to a matrix form where $\lambda_{p m}$ is the flux linkage of PM in the rotor and $\boldsymbol{i}_{f r}$ is the rotor field current. 
$V_{q d}=r_{s} i_{q d}+\frac{d}{d t} \lambda_{q d}+j \omega \lambda_{q d}$

$0=r_{r} i_{q d r}+\frac{d}{d t} \lambda_{q d r}+j\left(\omega-\omega_{r}\right) \lambda_{q d r}$

$V_{f r}=r_{f} i_{f r}+\frac{d}{d t} \lambda_{q d r}$

$\lambda_{q d r}=L_{r} i_{q d r}+L_{m}\left(i_{q d}+i_{f r}\right)+\lambda_{p m}$

In those equations, $\boldsymbol{i}_{q d}$ is the only measurable state variable and $\lambda_{q d r}$ is preferred as the stator variable because $\boldsymbol{i}_{q d r}$ is coupled with $\boldsymbol{i}_{q d}$ magnetically. From (24) and (26), $\boldsymbol{i}_{q d r}$ can be eliminated and the rotor state equation can be derived as (27).

$$
\begin{aligned}
\frac{d}{d t} \lambda_{\mathrm{qdr}} & =r_{r} L_{r}^{-1}\left[L_{m} i_{q d}+\left(L_{m} i_{f r}+\lambda_{p m}\right)\right. \\
& \left.-\left\{I+L_{r} r_{r}^{-1} j\left(\omega-\omega_{r}\right)\right\} \lambda_{q d r}\right]
\end{aligned}
$$

The flux linkage can be expressed as (28) where $L_{s}=L_{l s}+L_{m}$ and $L_{r}=L_{i r}+L_{m}$.

$$
\begin{aligned}
& \lambda_{q d}=L_{s} i_{q d}+L_{m}\left(i_{d r}+i_{f r}\right)+\lambda_{p m} \\
& \lambda_{q d r}=L_{r} i_{q d r}+L_{m}\left(i_{q d}+i_{f r}\right)+\lambda_{p m}
\end{aligned}
$$

Using (24), (26) and (28), the voltage equation is derived. From the voltage equation, the stator state equation becomes (29).

$$
\begin{aligned}
\frac{\mathrm{d}}{\mathrm{dt}} L_{s} \sigma i_{q d} & =V_{q d}-r_{s} i_{q d}-j \omega L_{s} \sigma i_{q d} \\
- & \left(+j \omega L_{m} L_{r}^{-1} \lambda_{q d r}+L_{m} L_{r}^{-1} \lambda_{q d r}\right) \\
& -\left(I-L_{m} L_{r}^{-1}\right)\left(\frac{\mathrm{d}}{\mathrm{dt}}+j \omega\right)\left(L_{m} i_{f r}+\lambda_{p m}\right)
\end{aligned}
$$

The difference between the IM model and IPMSM is thatIPMSM has permanent magnet. With these equations, the generalized $\mathrm{AC}$ machine model for an arbitrary reference frame can be induced. Finally (27) and (29) show the state differential equations. If an IM model is needed, the input current and flux linkage for IPMSM become 0 and the results would be same as those of previous models.

Fig. 6 shows the AC machine model block diagram made from (27) and (29). (4) is used for torque. It does not have cross coupling, has a simple structure and is well explained physically.

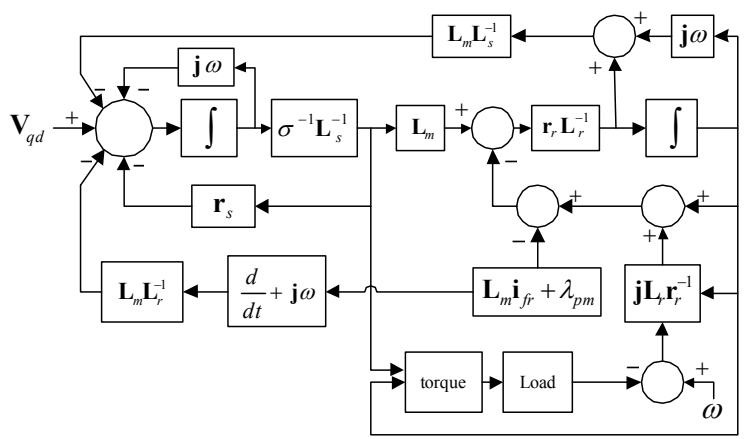

Fig. 6. Generalized AC machine model

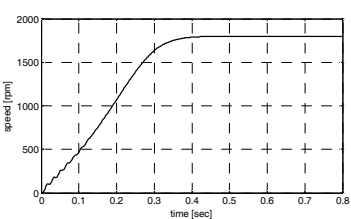

(a) speed response

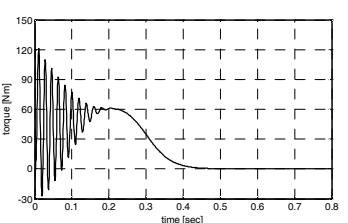

(b) torque response

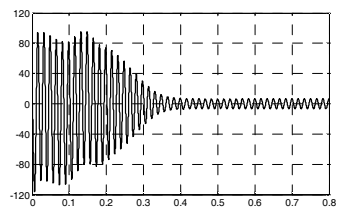

(c) current waveform of phase a

Fig. 7. IM waveform using matrix vector for stationary ref. frame

\section{Simulation and Comparison}

A simulation is performed for IM to compare the 
results with the other models. The applied voltage was $220 \mathrm{~V}, 60 \mathrm{~Hz}$. The specification of sample IM was that 4 poles, stator and rotor resistance are $0.435 \Omega, \quad 0.816 \Omega$, leakage and magnetization inductance are $2 \mathrm{mH}, 69.3 \mathrm{mH}$ each and moment of inertia is 0.089 . Fig. 7 shows the results of the IM for a matrix vector model and Ong's $\psi$ model. Because the sample is an IM, the field current and the flux linkage from PM become 0.

Fig. 7 (a) shows the speed response when the speed command is 1,800rpm. Fig. 7 (b) and 7 (c) show the torque response and phase acurrent waveform of an induction motor and the results are very similar to the results of other models [3] because the sample specification is the same. The block diagram shown in Fig. 6 has a simple structure and can be applied not only to IM but also to PMSM.

\section{Conclusions}

An AC machine model using a matrix vector with a d-qtransformation has been developed. It is a simple structure and useful for analyzing $\mathrm{AC}$ machines, being easy to apply to asymmetric machine of SM. The developed model can be used for both a synchronous and stationary reference frame because it is developed for an arbitrary frame. Future work to combine this model with the current controller model is desired and currently under way.

This work was supported by the National Research Foundation of Korea (NRF) grant funded by the Korea government(MEST) (No. 2010-0023043)

\section{References}

[1] Chee-Mun Ong, "Dynamic Simulation of Electric Machinery", Prenticel lall, 1997.

[2] K. Huh and R. D. Lorenz, "Discrete-Time Domain Modeling and Design for AC Machine Current Regulation," IEFE IAS Annual Meeting, pp.2066-2073, 2007.

[3] J. Holtz, Q. Juntao, J. Pontt, J. Rodriguez, P. Newman, and H. Miranda, "Design of fast and robust current regulators for high-power drives based on complex state variables," Industry Applications, IIII Transactions on, vol. 40, pp. 1388-1397, 2004.

[4] H. Kim and R. D. Lorenz, "Synchronous frame PI current regulators in a virtually translated system," presented at 2004 IIII IAS Annual Meeting, Seattle, WA, USA, 2004.

[5] D. W. Novotny and T. A. Lipo, Vector Control and Dynamics of AC Drives : Clarendon Press Oxford 1996.

[6] S. Hong, "A Study on AC Machine Modeling using Complex Vector and $\mathrm{d}-\mathrm{q}$ Transformation", KIII Transactions on, vol. 61, No.11, pp.1601-1605, 2013.

\section{저자소개}

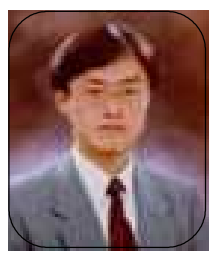

Sun-Ki Hong

Sun-Ki Hong graduated from the Department of Electrical Engineering, Seoul National University in 1987. He received his M.S. and $\mathrm{PhD}$ in electrical engineering from Seoul National University in 1989 and 1993, respectively. He became a Member of the IEEE in 1993and worked as a researcher at REX Industrial Co., Ltd. from 1993 to 1995. He has been teaching at the Department of Digital Control Engineering, Hoseo University since 1995. His special interests include the modeling and computation of hysteresis in electro-magnetic system; present interests are the fields of design and analysis of electric machine with finite element methods and power converter and inverter etc. 